\title{
Positive solutions of a renewal equation
}

\author{
by JANusz TRAPLE (Kraków)
}

\begin{abstract}
An existence theorem is proved for the scalar convolution type integral equation $x(t)=\int_{-\infty}^{\infty} h(t-s) f(s, x(s)) d s$.
\end{abstract}

Introduction. In the paper [1] K. L. Cooke and J. L. Kaplan initiated theoretical analysis of the equation

$$
x(t)=\int_{-\infty}^{\infty} h(t-s) f(s, x(s)) d s, \quad t \in \mathbb{R},
$$

where $h(s)=1_{[0, \tau]}(s)$, as a model for some infectious diseases, or as the growth equation for a single species population. They proved under the assumptions of periodicity and continuity of $f$ that if $\tau$ is large enough, then there exists a positive periodic solution of (1) with period equal to the period of $f$.

In this paper we study the problem of existence of a nonnegative nontrivial solution to (1) in a general situation when $h \in L_{+}^{1}$ and $f$ is a continuous function. This problem is much more mathematically delicate than the periodic one, because the operator given by the right hand side of (1) is not completely continuous in the Banach space of all continuous and bounded functions on $\mathbb{R}$ with the supremum norm. So our methods are quite different from those of Cooke and Kaplan. In the last part of the paper we prove the existence of a nonnegative, nontrivial, periodic solution to (1) whenever $f$ is a periodic, continuous function and $h \in L_{+}^{1}$.

The periodic case has been considered independently in an unpublished paper of P. Kasprowski [2], but our proofs are quite different. Similar biological models have also been studied by Swick [4] and London and Yorke [3].

1991 Mathematics Subject Classification: Primary 45M20; Secondary 92D25.

Key words and phrases: integral equations, convolution. 
The existence theorem. We consider a convolution type, scalar integral equation of the form (1), where $h$ is a nonnegative, integrable function on the real line $\mathbb{R}$ and $f$ is a scalar-valued, continuous function defined on $\mathbb{R} \times[0, \infty)$. We study the problem of the existence of nonnegative, continuous, bounded, not identically zero solutions of (1).

In the paper $C$ denotes the locally convex topological vector space of all real-valued, continuous functions on $\mathbb{R}$, equipped with the topology of uniform convergence on compact sets. We define $C_{+}=\{z \in C: z(t) \geq 0$ for all $t \in \mathbb{R}\}$.

The following theorems are the main results of the paper:

THEOREM 1. Assume that there exist positive constants $M, k, \varepsilon$ such that

$$
\begin{gathered}
0 \leq f(t, x) \leq M, \quad \text { for } t \in \mathbb{R}, x \in[0, \infty), \\
f(t, x) \geq k x, \quad \text { for } t \in \mathbb{R}, x \in[0, \varepsilon), \\
\quad k \int_{-\infty}^{\infty} h(s) d s>1 .
\end{gathered}
$$

Then there exists a nontrivial bounded solution $x \in C_{+}$of (1). Moreover, there exists a constant $\lambda=\lambda(h, k, \varepsilon)>0$ such that on any interval $[\alpha, \beta]$ with $\beta-\alpha \geq \lambda$,

$$
\sup \{x(t): \alpha \leq t \leq \beta\}>\varepsilon .
$$

TheOREM 2. Assume that the hypotheses of Theorem 1 are satisfied. Then for any nontrivial solution $x \in C_{+}$of (1) the following statements are true:

(i) $\inf \{t: x(t) \geq \varepsilon\}=: t_{1}(x)<t_{2}(x):=\sup \{t: x(t) \geq \varepsilon\}$,

(ii) if $k \int_{0}^{\infty} h(s) d s>1$ (resp. $k \int_{-\infty}^{0} h(s) d s>1$ ), then $t_{2}(x)=\infty$ (resp. $\left.t_{1}(x)=-\infty\right)$,

(iii) if $t_{2}(x)<\infty$ (resp. $\left.t_{1}(x)>-\infty\right)$, then $x(t) \rightarrow 0$ as $t \rightarrow \infty$ (resp. $t \rightarrow-\infty)$.

Moreover, there exists a constant $\lambda=\lambda(h, k, \varepsilon)>0$ such that for every nontrivial solution $x \in C_{+}$of (1) inequality (5) holds, provided $t_{1}(x) \leq \alpha<$ $\beta \leq t_{2}(x), \beta-\alpha \geq \lambda$.

Before giving the proofs of the theorems we establish some results concerning convolution type inequalities. The next two lemmas play a crucial role in the proofs of both theorems.

Let $L^{1}, L_{+}^{1},\|\|_{1}$ denote, respectively, the Banach space of all integrable functions on $\mathbb{R}$, the set of all nonnegative elements of $L^{1}$ and the $L^{1}$ norm. 
LEMma 1. Assume that $g \in L_{+}^{1}$ and $\|g\|_{1}>1$. Then $x=0$ is the unique bounded, continuous, nonnegative solution of the inequality

$$
x(t) \geq \int_{-\infty}^{\infty} g(t-s) x(s) d s, \quad t \in \mathbb{R} .
$$

Proof. For $c>0$, set $\eta^{c}(s)=\exp (-c|s|), s \in \mathbb{R}$. Since $\|g\|_{1}>1$, there exists $a>0$ such that also $\left\|g \eta^{a}\right\|_{1}>1$. From (6) and $\eta^{a}(t) \geq \eta^{a}(t-s) \eta^{a}(s)$ for $s, t \in \mathbb{R}$, we obtain

$$
\left\|x \eta^{a}\right\|_{1} \geq \int_{-\infty}^{\infty} \int_{-\infty}^{\infty} g(t-s) \eta^{a}(t-s) x(s) \eta^{a}(s) d s d t=\left\|g \eta^{a}\right\|_{1}\left\|x \eta^{a}\right\|_{1} .
$$

Therefore $\left\|x \eta^{a}\right\|_{1}=0$ and in consequence $x=0$.

For $x \in C, \omega_{x}$ denotes the modulus of continuity of $x$, i.e. $\omega_{x}(\delta)=$ $\sup \left\{\left|x\left(t^{\prime}\right)-x(t)\right|: t, t^{\prime} \in \mathbb{R},\left|t-t^{\prime}\right| \leq \delta\right\}, \delta \geq 0$. If $m$ is a function, $\omega_{x} \leq m$ means $\omega_{x}(\delta) \leq m(\delta)$ for every $\delta \geq 0$.

Lemma 2. Let $g \in L_{+}^{1}$ with $\|g\|_{1}>1$, let $\varepsilon>0$, and let $m:[0, \infty) \rightarrow$ $[0, \infty)$ be a nondecreasing function, continuous at 0 , with $m(0)=0$. Then for any uniformly continuous nontrivial solution $x \in C_{+}$of the inequality

$$
x(t) \geq \int_{-\infty}^{\infty} g(t-s) 1_{[0, \varepsilon]}(x(s)) x(s) d s, \quad t \in \mathbb{R},
$$

the following statements are true:

(8) $\quad \inf \{t: x(t) \geq \varepsilon\}=: t_{1}(x)<t_{2}(x):=\sup \{t: x(t) \geq \varepsilon\}$,

(9) if $\int_{0}^{\infty} g(s) d s>1\left(\right.$ resp. $\left.\int_{-\infty}^{0} g(s) d s>1\right)$, then $t_{2}(x)=\infty$ (resp. $\left.t_{1}(x)=-\infty\right)$,

(10) if $t_{2}(x)<\infty$ (resp. $\left.t_{1}(x)>-\infty\right)$, then $x(t) \rightarrow 0$ as $t \rightarrow \infty$ (resp. $t \rightarrow-\infty)$.

Moreover, there exists a constant $\lambda=\lambda(m, g, \varepsilon)>0$ such that if additionally $\omega_{x} \leq m$, then

(11) $\sup \{x(t): \alpha \leq t \leq \beta\}>\varepsilon \quad$ for each $\alpha, \beta$, where $\beta-\alpha \geq \lambda$.

Proof. Since $\|g\|_{1}>1$, there exists $l_{0}>0$ such that

$$
b:=\int_{-l_{0}}^{l_{0}} g(s) d s>1
$$

First of all we establish the following three properties:

Property A. For every $c>0$, there exists $l>0$ such that if $z \in C_{+}$ satisfies (7) and $\varepsilon \geq z(t) \geq c$ for $t \in[\alpha, \beta]$, then $\beta-\alpha<l$. 
Easy computation shows that $l:=2 \bar{n} l_{0}$, where $\bar{n}$ is a natural number such that $b^{\bar{n}} c>\varepsilon$, satisfies the assertion of Property A.

Property B. For every $d>0$ and $\gamma>0$, there exist $l>0$ and $c>0$ with $l \geq d$ such that

(i) if $\int_{0}^{l_{0}} g(s) d s>0$ and if $z \in C_{+}$satisfies $(7), z(t) \leq \varepsilon$ for $t \in\left[\alpha^{\prime}, \beta^{\prime}\right]$ where $\beta^{\prime}-\alpha^{\prime} \geq l+l_{0}$ and $z(t) \geq \gamma$ for $t \in\left[\alpha^{\prime}, \alpha^{\prime}+d\right]$, then $z(t)>0$ for $t \in\left[\alpha^{\prime}+l, \beta^{\prime}\right]$ and $z(t) \geq c$ for $t \in\left[\alpha^{\prime}+l, \alpha^{\prime}+l+l_{0}\right]$,

(ii) if $\int_{-l_{0}}^{0} g(s) d s>0$ and if $z \in C_{+}$satisfies $(7), z(t) \leq \varepsilon$ for $t \in\left[\alpha^{\prime}, \beta^{\prime}\right]$ where $\beta^{\prime}-\alpha^{\prime} \geq l+l_{0}$ and $z(t) \geq \gamma$ for $t \in\left[\beta^{\prime}-d, \beta^{\prime}\right]$, then $z(t)>0$ for $t \in\left[\alpha^{\prime}, \beta^{\prime}-l\right]$ and $z(t) \geq c$ for $t \in\left[\beta^{\prime}-l-l_{0}, \beta^{\prime}-l\right]$.

In case (i) there exist $\bar{t}>0, r>0, p>0$ such that $r \leq \bar{t} \leq l_{0}, 4 r \leq d$, $p \leq 1$ and

$$
\int_{\bar{t}-2 r}^{\bar{t}-r} g(s) d s \geq p, \quad \int_{\bar{t}+r}^{\bar{t}+2 r} g(s) d s \geq p .
$$

Let $\bar{n}$ be a natural number such that $d+2 \bar{n} r \geq l_{0}$, and let $\beta^{\prime}-\alpha^{\prime} \geq l+l_{0}$, where $l:=\bar{n} \bar{t}-\bar{n} r$. Since $z$ satisfies (7), easy computation shows that

$$
z(t) \geq \gamma \int_{\alpha^{\prime}}^{\alpha^{\prime}+d} g(t-s) d s \geq \gamma p
$$

for $t \in\left[\alpha^{\prime}+\bar{t}-r, \alpha^{\prime}+d+\bar{t}-r\right]$. Using this argument $\bar{n}$ times we obtain $z(t) \geq \gamma p^{\bar{n}}=: c$ for $t \in\left[\alpha^{\prime}+l, \alpha^{\prime}+l+l_{0}\right]$. Continuing we conclude that $z(t)>0$ on the whole interval $\left[\alpha^{\prime}+l, \beta^{\prime}\right]$, because $\bar{t} \leq l_{0}$. The proof of (ii) is similar.

Property C. Assume that $z \in C_{+}$satisfies (7) and $0<z(t) \leq \varepsilon$ for $t \in\left[\alpha^{\prime \prime}, \beta^{\prime \prime}\right]$, where $\beta^{\prime \prime}-\alpha^{\prime \prime} \geq 2 l_{0}$. Assume moreover that either

(i) $z(t) \geq \gamma>0$ for $t \in\left[\alpha^{\prime \prime}, \alpha^{\prime \prime}+l_{0}\right] \cup\left[\beta^{\prime \prime}-l_{0}, \beta^{\prime \prime}\right]$ and $\int_{-l_{0}}^{0} g(s) d s<1$, $\int_{0}^{l_{0}} g(s) d s<1, \int_{-l_{0}}^{l_{0}} g(s) d s>1$, or

(ii) $z(t) \geq \gamma$ for $t \in\left[\alpha^{\prime \prime}, \alpha^{\prime \prime}+l_{0}\right]$ and $\int_{0}^{l_{0}} g(s) d s>1$, or

(iii) $z(t) \geq \gamma$ for $t \in\left[\beta^{\prime \prime}-l_{0}, \beta^{\prime \prime}\right]$ and $\int_{-l_{0}}^{0} g(s) d s>1$.

Then $z(t) \geq \gamma$ for all $t \in\left[\alpha^{\prime \prime}, \beta^{\prime \prime}\right]$.

To prove this assume (i) holds. Suppose that $z\left(t_{0}\right)$ is the minimal value of $z$ on $\left[\alpha^{\prime \prime}, \beta^{\prime \prime}\right]$. Then $t_{0} \in\left[\alpha^{\prime \prime}+l_{0}, \beta^{\prime \prime}-l_{0}\right]$ would imply by $(7)$ that $z\left(t_{0}\right) \geq z\left(t_{0}\right) \int_{\alpha^{\prime \prime}}^{\beta^{\prime \prime}} g\left(t_{0}-s\right) d s>z\left(t_{0}\right)$, which is impossible. Thus we must have $t_{0} \in\left[\alpha^{\prime \prime}, \alpha^{\prime \prime}+l_{0}\right] \cup\left[\beta^{\prime \prime}-l_{0}, \beta^{\prime \prime}\right]$ and in consequence $z(t) \geq \gamma$ for all $t \in\left[\alpha^{\prime \prime}, \beta^{\prime \prime}\right]$. The proofs in the remaining cases are similar. 
After these preparations, assume now that $x \in C_{+}$is a nontrivial solution of (7). By Lemma 1 it is impossible to have $x(t) \leq \varepsilon$ for all $t \in \mathbb{R}$. Hence the quantities $t_{1}(x)$ and $t_{2}(x)$ in $(8)$ are well defined and $t_{1}(x)<t_{2}(x)$.

In order to prove (9) assume that $\int_{0}^{\infty} g(s) d s>1$ and suppose contrary to (9) that $\alpha=t_{2}(x)<\infty$. Then $x(t)<\varepsilon$ for all $t>\alpha$. Since $x$ is continuous and $x(\alpha)=\varepsilon$, there exists $d_{1}>0$ such that $x(t) \geq \varepsilon / 2$ for $t \in\left[\alpha, \alpha+d_{1}\right]$. By Property B there exist $l_{1}=l\left(\varepsilon / 2, d_{1}\right)$ and $c_{1}=c\left(\varepsilon / 2, d_{1}\right)$ such that $x(t) \geq c_{1}$ for $t \in\left[\alpha+l_{1}, \alpha+l_{1}+l_{0}\right]$ and $x(t)>0$ for all $t \geq \alpha+l_{1}$. Hence by Property $\mathrm{C}$ we obtain $x(t) \geq c_{1}$ for all $t \geq \alpha+l_{1}$. But this is impossible by Property A. The proof in the second case is similar.

We will now prove (11). Let $\bar{d}$ be a constant such that $m(\bar{d}) \leq \varepsilon / 2$ and let $\bar{l}$ and $\bar{c}$ be the quantities given by Property $\mathrm{B}$ with $\gamma=\varepsilon / 2$ and $d=\bar{d}$. Suppose that $x(t) \leq \varepsilon$ for $t \in[\alpha, \beta]$, where $\beta-\alpha \geq 2 \bar{l}+2 l_{0}$ and $x(\alpha)=x(\beta)=\varepsilon$. The assumption $\omega_{x} \leq m$ implies $x(t) \geq \varepsilon / 2$ for $t \in[\alpha, \alpha+\bar{d}] \cup[\beta-\bar{d}, \beta]$. Consider now the following three cases: (i) $a_{1}:=\int_{0}^{l_{0}} g(s) d s<1$ and $a_{2}:=\int_{-l_{0}}^{0} g(s) d s<1$, (ii) $a_{1}>1$, and (iii) $a_{2}>1$.

In the first case from (12) it follows that $a_{1}>0$ and $a_{2}>0$ and by the definition of $\bar{l}$ and $\bar{c}$ we get $x(t) \geq \bar{c}$ for $t \in\left[\alpha+\bar{l}, \alpha+\bar{l}+l_{0}\right] \cup\left[\beta-\bar{l}-l_{0}, \beta-\bar{l}\right]$ and $x(t)>0$ for all $t \in[\alpha+\bar{l}, \beta-\bar{l}]$. Therefore Property $\mathrm{C}$ yields the estimate $x(t) \geq \bar{c}$ for all $t \in[\alpha+\bar{l}, \beta-\bar{l}]$. Similar reasoning shows that the same estimate is true in the remaining cases. Hence if $\beta-\alpha \geq \lambda:=$ $\max \left\{2 \bar{l}+2 l_{0}, l(\bar{c})+2 \bar{l}\right\}$, where $l(\bar{c})$ is given by Property A with $c=\bar{c}$, then (11) is satisfied.

Now we prove (10) in the case $t_{2}(x)<\infty$. We have $x(t)<\varepsilon$ for all $t>t_{2}(x)$. Suppose contrary to $(10)$ that $\lim _{\sup _{t \rightarrow \infty}} x(t)=\delta>0$. Fix $\delta^{\prime}$ with $0<\delta^{\prime}<\delta$. Since $x$ is uniformly continuous, there exist $d^{\prime}>0$ and a sequence of intervals $\left[\alpha_{n}, \beta_{n}\right]$ with $\beta_{n}-\alpha_{n} \geq d^{\prime}, n=1,2, \ldots, \alpha_{n} \rightarrow \infty$, such that $x(t) \geq \delta^{\prime}$ for $t \in\left[\alpha_{n}, \beta_{n}\right], n=1,2, \ldots$ Now the same reasoning as in the proof of (11) shows the existence of some $l_{1}=l\left(\delta^{\prime}, d^{\prime}\right)>0$ and $c_{1}=c\left(\delta^{\prime}, d^{\prime}\right)>0$ such that $x(t) \geq c_{1}$ for all $t \geq \alpha_{1}+l_{1}$. But by Property A this is impossible and thus $\delta=0$. The proof of (10) in the case $t_{1}(x)>-\infty$ is similar.

Proof of Theorem 1. Besides equation (1) we consider the family of equations

$$
x(t)=\int_{-\infty}^{\infty} h(t-s) f(s, x(s)) d s+\sigma=: T_{\sigma}(x)(t), \quad t \in \mathbb{R},
$$

where $\sigma \in(0,1)$ is a constant. It is easily seen that each $T_{\sigma}$ is a continuous 
transformation from $C_{+}$into $C_{+}$. For every $x \in C_{+}$and $\sigma$ we have

$$
\begin{gathered}
\left|T_{\sigma}(x)(t+s)-T_{\sigma}(x)(t)\right| \leq M \int_{-\infty}^{\infty}|h(r+s)-h(r)| d r=: m_{0}(s), \\
\sigma \leq T_{\sigma}(x)(t) \leq M \int_{-\infty}^{\infty} h(s) d s+1=: M_{0}
\end{gathered}
$$

for each $t, s \in \mathbb{R}$. Relations (14) and (15) guaranteed by the Arzelà-Ascoli theorem, $T_{\sigma}$ has values in a compact, convex subset $D$ of $C_{+}$, for each $\sigma$. Hence by the Tikhonov fixed point theorem, for each $\sigma$ there exists a solution $x_{\sigma} \in D$ of equation (13). Assumption (3) implies that the $x_{\sigma}$ are solutions of the inequality

$$
x(t) \geq \int_{-\infty}^{\infty} k h(t-s) 1_{[0, \varepsilon]}(x(s)) x(s) d s, \quad t \in \mathbb{R} .
$$

Therefore Lemma 2 with $g(s)=k h(s), m(s)=\sup \left\{m_{0}(\bar{s}): 0 \leq \bar{s} \leq s\right\}$ implies that there exists $\lambda>0$ such that

$$
\sup \left\{x_{\sigma}(t): \alpha \leq t \leq \beta\right\}>\varepsilon
$$

for all $\alpha, \beta \in \mathbb{R}$ with $\beta-\alpha \geq \lambda$ and all $\sigma \in(0,1)$. Since all $x_{\sigma}$ belong to a compact set $D$, we can find a subsequence of $\left\{x_{\sigma}\right\}$ which converges to a function $x \in C_{+}$as $\sigma \rightarrow 0$. Of course $x$ is a solution of (1) and by (17), $x$ is nontrivial. Since $x$ is also a solution of (16), by Lemma 2 inequality (5) also holds. This completes the proof of Theorem 1.

Proof of Theorem 2. The assertion of Theorem 2 follows immediately from that of Lemma 2 and the fact that under our assumptions each solution of (1) is also a solution of (16).

The periodic theorem. In this section we consider equation (1) under the additional assumption that $f$ is periodic. We suppose that $f(t+\omega, x)=$ $f(t, x)$ for all $t \in \mathbb{R}, x \in[0, \infty)$. The following theorem is a generalization of Theorem 1 of [1].

TheOREM 3. Assume the hypotheses of Theorem 1 . If $f$ is an $\omega$-periodic function, then equation (1) has an w-periodic, nontrivial solution.

Proof. From the proof of Theorem 1 it follows immediately that if $G$ is a closed, convex subset of $C$ and $T_{\sigma}(G) \subset G$ for each $\sigma>0$, then the solution given by Theorem 1 belongs to $G$. Therefore, since under our assumptions the set of all $\omega$-periodic functions satisfies the above condition, the solution given by Theorem 1 is an $\omega$-periodic function. 
Acknowledgments. The author thanks Prof. A. Lasota for helpful discussions and P. Kasprowski for showing his unpublished paper.

\section{References}

[1] K. L. Cooke and J. L. Kaplan, A periodicity threshold theorem for epidemics and population growth, Math. Biosci. 31 (1976), 87-104.

[2] P. Kasprowski, On positive solutions of nonlinear convolution equations, unpublished paper.

[3] W. P. London and J. A. Yorke, Recurrent outbreaks of measles, chickenpox and mumps. II. Systematic differences in contact rates and stochastic effects, Amer. J. Epidemiol. 98 (1973), 469-482.

[4] K. E. Swick, A model of single species population growth, SIAM J. Math. Anal. 7 (1976), 565-576.

INSTITUTE OF INFORMATICS

JAGIELLONIAN UNIVERSITY

NAWOJKI 11

30-072 KRAKÓW, POLAND 